\title{
A giant galaxy in the young Universe with a massive ring
}

Tiantian Yuan ${ }^{1,2, *}$, Ahmed Elagali ${ }^{3,2}$, Ivo Labbé ${ }^{1}$, Glenn G. Kacprzak ${ }^{1,2}$, Claudia del P. Lagos ${ }^{3,2,4}$, Leo Y. Alcorn ${ }^{5,6}$, Jonathan H. Cohn ${ }^{5}$, Kim-Vy H. Tran ${ }^{5,7,2}$, Karl Glazebrook ${ }^{1,2}$, Brent A. Groves ${ }^{3,8,2}$, Kenneth C. Freeman ${ }^{8}$, Lee R. Spitler ${ }^{9,10,2}$, Caroline M. S. Straatman ${ }^{11}$, Deanne B. Fisher ${ }^{1,2}$, Sarah M. Sweet ${ }^{1,2,12}$

\begin{abstract}
${ }^{1}$ Centre for Astrophysics and Supercomputing, Swinburne University of Technology, Hawthorn, Victoria 3122, Australia. ${ }^{2}$ ARC Centre of Excellence for All Sky Astrophysics in 3 Dimensions (ASTRO 3D), Australia. ${ }^{3}$ International Centre for Radio Astronomy Research (ICRAR), M468, The University of Western Australia, 35 Stirling Highway, Crawley, WA 6009, Australia. ${ }^{4}$ Cosmic Dawn Center (DAWN), Denmark. ${ }^{5}$ George P. and Cynthia Woods Mitchell Institute for Fundamental Physics and Astronomy, Department of Physics \& Astronomy, Texas A\&M University, College Station, TX, 77843, USA. ${ }^{6}$ Department of Physics and Astronomy, York University, 4700 Keele St., Toronto, Ontario, Canada, MJ3 1P3. ${ }^{7}$ School of Physics, University of New South Wales, Sydney, NSW 2052, Australia. ${ }^{8}$ Research School of Astronomy and Astrophysics, the Australian National University, Canberra, ACT 2611, Australia. ${ }^{9}$ Research Centre for Astronomy, Astrophysics \& Astrophotonics, Macquarie University, Sydney, NSW 2109, Australia. ${ }^{10}$ Department of Physics \& Astronomy, Macquarie University, Sydney, NSW 2109, Australia. ${ }^{11}$ Sterrenkundig Observatorium, Universiteit Gent, Krijgslaan 281 S9, 9000 Gent, Belgium. ${ }^{12}$ School of Mathematics and Physics, University of Queensland, Brisbane, Qld 4072, Australia
\end{abstract}

In the local $(z \approx 0)$ Universe, collisional ring galaxies make up only $\sim 0.01 \%$ of galaxies ${ }^{11}$ and are formed by head-on galactic collisions that trigger radially propagating density waves 24 . These striking systems provide key snapshots for dissecting galactic disks and are studied extensively in the local Universe ${ }^{5.9}$. However, not much is known about distant $(z>0.1)$ collisional rings $\frac{10 \cdot 14}{\text {. Here we present }}$ a detailed study of a ring galaxy at a look-back time of $10.8 \mathrm{Gyr}$ $(z=2.19)$. Compared with our Milky Way, this galaxy has a similar stellar mass, but has a stellar half-light radius that is $1.5-2.2$ times larger and is forming stars 50 times faster. The extended, diffuse stellar light outside of the star-forming ring, combined with a radial velocity on the ring and an intruder galaxy nearby, provides evidence for this galaxy hosting a collisional ring. If the ring is secularly evolved $^{15 ; 16}$, the implied large bar in a giant disk would be inconsistent with the current understanding of the earliest formation of barred spirals $s^{17.21}$. Contrary to previous predictions $s^{10 \cdot 12}$, this work suggests that massive collisional rings were as rare 11 Gyr ago as they are today. Our discovery offers a unique pathway for studying density waves in young galaxies, as well as constraining the cosmic evolution of spiral disks and galaxy groups.

The ring galaxy (ID 5519, hereafter R5519) was discovered in our systematic search for $z \gtrsim 2$ spiral galaxies in the Cosmic Evolution Survey (COSMOS) field of the FourStar Galaxy Evolution survey (ZFOURGE ${ }^{22}$ ). We used ZFOURGE catalogue images to identify spiral structures in galaxies within the photometric redshift range of $1.8 \lesssim z_{p} \lesssim 2.5$. Owing to the surface brightness dimming and smaller sizes of galaxies at $z>1$, our visual identification of spiral features was restricted to galaxies with illuminated pixels larger than a radius of 0.15 ( $>4 \mathrm{kpc}$ at $z \approx 2$ ) in the Hubble Space Telescope (HST) images. Our visual inspection simultaneously identified ring galaxies and other morphologically distinct objects such as mergers and gravitationally lensed galaxies. R5519 was flagged as one of the largest galaxies among the $\sim 4000$ galaxies inspected, with a clear ring structure as well as a large diffuse disk (Fig.1 and Supplementary Figs.1-2).

We confirm the spectroscopic redshift of R5519 to be $z_{s}=$ $2.192 \pm 0.001$ based on our Keck/MOSFIRE near-infrared (NIR) spectroscopy and Keck/OSIRIS adaptive-optics aided NIR integral field spectroscopy (Supplementary Figs.3-4). A joint analysis of the MOSFIRE and OSIRIS spectroscopic data, in combination with the groundbased $\mathrm{H} \alpha$ narrow-band image from the ZFOURGE catalogue, shows that the $\mathrm{H} \alpha$ kinematics are consistent with a tilted rotating and expanding/contracting circular ring model (Fig.2, Methods). Taking the inclination angle $\left(i=29^{\circ} \pm 5\right)$ and the position angle $\left(P A=28^{\circ} \pm 10\right)$ from an ellipse fit to the ring morphology (Supplementary Table 1, Supplementary Fig.1) as inputs to the kinematic model, the inferred rotational velocity at the fixed ring radius $\left(\mathrm{R}_{\text {ring }}=5.1 \pm 0.4 \mathrm{kpc}\right)$ is $\mathrm{V}_{\text {rot }}=90$ $\pm 75 \mathrm{~km} / \mathrm{s}$ and the radial expansion/contraction velocity is $\mathrm{V}_{\text {rad }}=226$ $\pm 90 \mathrm{~km} / \mathrm{s}$. The velocity error bars represent uncertainties from observational measurements. The systematic errors caused by uncertain ranges of $P A$ and $i$ are of the same order of magnitude (Supplementary Figs.5-9).

We verify that R5519 resides in a small galaxy group environment, reminiscent of the loose groups in which local collisional ring galaxies (CRGs) such as the Cartwheel galaxy are found 14/5. A companion galaxy (ID 5593, hereafter G5593) is confirmed at a projected distance of $\sim 30 \mathrm{kpc}$ from R5519 with a 3D-HST survey ${ }^{23}$ grism redshift of $z_{\text {grism }}=2.184_{-0.006}^{+0.005}$ (Fig.1). An additional group candidate (ID 5475, hereafter G5475) is found at a projected distance of $\sim 40 \mathrm{kpc}$ away (Fig.1), with a photometric redshift ${ }^{222}$ of $z_{p}=2.1 \pm 0.1$. If G5593 is the intruder of R5519, then the inferred timescale after collision is $\tau_{c}>39_{-15}^{+65} \mathrm{Myr}$; this is a lower limit due to unknown projection effects.

R5519 has a total UV+IR star formation rate (SFR) of $80.0 \pm 0.2$ $\mathrm{M}_{\odot} \mathrm{yr}^{-1}$ and a stellar mass of $\log \left(\mathrm{M}_{*} / \mathrm{M}_{\odot}\right)=10.78 \pm 0.03$. In comparison, G5593 has a total SFR of $123 \pm 2 \mathrm{M}_{\odot} \mathrm{yr}^{-1}$ and $\log \left(\mathrm{M}_{*} / \mathrm{M}_{\odot}\right)=$ $10.40 \pm 0.04$ (Table 1, Methods). The furthest group candidate (G5475) within a $50 \mathrm{kpc}$ projected distance from the ring is a compact quiescent galaxy. The morphology of G5593 shows double nuclei and a tidal tail (Fig.1), suggestive of an ongoing merger of its own. We find no active galactic nucleus (AGN) signatures in R5519 nor in its companions based on the ZFOURGE AGN catalogue ${ }^{24}$ and our MOSFIRE spectrum. The derived interstellar medium (ISM) properties indicate that R5519 is metal-rich $(12+\log (\mathrm{O} / \mathrm{H})=8.6-8.8)$ (Supplementary Information, Supplementary Fig.3).

R5519's ring has the highest contrast in HST F125W and F160W images (Fig.1). At $z_{s}=2.19, \mathrm{~F} 125 \mathrm{~W}$ and F160W filters include contributions from strong emission lines such as [OII] $\lambda 3727$ and $[\mathrm{OIII}] \lambda 5007$, respectively. The prominent ring structure in these bands 
is consistent with the ring being dominated by emission from luminous star-formation regions. The ground-based $\mathrm{H} \alpha$ narrow-band image suggests that the bulk ( $45-70 \%)$ of recent star formation occurs on the ring (Methods). We find tentative evidence for the existence of an offcentre "nucleus" based on the redder colour of one spatially resolved region in the deep $\mathrm{Ks}_{\text {tot }}$ and HST near-infrared images (Methods, Supplementary Fig.2, Supplementary Table 2). For a range of extreme star-formation histories (10 Myr burst and constant SFR), we derive for R5519 a total stellar mass between $10^{10.5}-10^{10.8} \mathrm{M}_{\odot}$, and stellar ages between 0.05-2 Gyr (Methods, Supplementary Fig.10).

The ring of R5519 is small compared with local CRGs. For example, $90 \%$ of rings in the local CRG sample ${ }^{1}$ have ring radius $\gtrsim 5$ kpc. R5519's SFR is at least $4 \times$ larger than local CRGs $\sqrt{25}$ of similar stellar masses (Table 1, Supplementary Fig.11). The enhanced SFR of R5519 compared with local CRGs is understandable in the context that high- $z$ star-forming galaxies have a larger molecular gas fraction 26 . The average star formation rate surface density $\left(\Sigma_{\mathrm{SFR}}\right)$ of $\mathrm{R} 5519$ is $\sim 0.3 \mathrm{M}_{\odot} \mathrm{yr}^{-1} \mathrm{kpc}^{-1}$, typical of a star-forming galaxy at $z \approx 2$, and $\sim 4-8$ times larger than local CRGs such as Arp 147 and the Cartwhee 9.27 . Interestingly, nearby CRGs show moderately elevated SFR relative to $z \sim 0$ isolated disks ${ }^{25}$, whereas R5519 does not have a substantially higher SFR in relation to its $z \approx 2$ peers (Supplementary Fig.11). Both R5519 and its companion G5593 lie within the 0.3 dex scatter of the $\mathrm{M}_{*}$-SFR "main-sequence' ${ }^{28}$ relation of star-forming galaxies at $z \approx 2$. CRGs are rare laboratories to study star formation in interacting galaxies 49 . Future observations on the molecular gas would be important in revealing the details of the star formation processes in R5519.

One of the most striking features of R5519 is the extended stellar light outside of the ring in multiple wavelengths (Fig.1, Supplementary Fig.2). We have ruled out R5519 as a regular merger or a gravitationally lensed system (see Supplementary Information). We quantify the size of the diffuse light by measuring $\mathrm{R}_{80}$, the radius within which $80 \%$ of the total luminosity is included (Methods, Table 1, Supplementary Figs.12-13). Comparing with other $z \sim 2$ galaxies in the 3D-HST catalogue ${ }^{29}$, R5519's $\mathrm{R}_{80}$ is $2.4 \sigma$ larger than the mean size $(5.4 \mathrm{kpc})$ of all late-type galaxies $\left(\log \left(\mathrm{M}_{*} / \mathrm{M}_{\odot}\right)>9.5\right)$ and $1.5 \sigma$ larger than the mean value $(7.1 \mathrm{kpc})$ of the most massive $\left(\log \left(\mathrm{M}_{*} / \mathrm{M}_{\odot}\right) \geq 10.6\right)$ latetype galaxies at $z \approx 2$ (Fig.3). A morphological inspection on the other unusually large ( $1 \sigma$ above the mean) and massive late-type galaxies in the COSMOS field reveals that most (4/7) of them are probably mergers (Fig.3). Excluding the 4 mergers, the mean value of the most massive galaxies at $z \approx 2$ is $6.4 \mathrm{kpc}$ and is $2.6 \sigma$ lower than R5519. Compared with our Milky Way's stellar disk, the half-light radius of R5519 is 1.5-2.2 times larger and its $\mathrm{R}_{80}$ is 1.2-1.8 times larger (Methods).

If R5519 is a secularly evolved resonant ring ${ }^{15}$ (see Supplementary Information), then the giant disk and the implied large bar (half length $\sim 5 \mathrm{kpc}$, similar to the Milky Way's bar) is challenging to understand at this redshift. Diffuse stellar disks and/or bars as large as those of R5519 have not been conclusively reported in observations or simulations at $z>2$ [ref $\left.\frac{14|17| 18,20}{2}\right]$. For the rare, smaller ( $<1 \mathrm{kpc}$ in radius) barred spiral galaxies formed in simulations at $z>2$ [ref $\left[^{17,19,21}\right]$, they are relatively isolated and do not reside in an active environment like R5519. Our interpretation of a collisional ring instead of a secularly evolved ring can be verified by high spatial resolution imaging with the James Webb Space Telescope (JWST) in the mid-IR wavelength.

If R5519 is exhibiting a first ring after collision in the classic model of an expanding wave 4 [6.8, the large $\mathrm{R}_{\text {disk }}$ and small $\mathrm{R}_{\text {ring }}$ imply different collisional timescales $\left(\tau_{c} \geq 80 \mathrm{Myr}\right.$ and $\tau_{c} \leq 50 \mathrm{Myr}$, see Supplementary Information). The inconsistency in $\tau_{c}$ can be reconciled if the current ring in R5519 is a second ring after the collision. A local analogy would be the Cartwheel galaxy 5 ,9 , when Cartwheel's outer ring fades and the inner second ring dominates. A second ring would explain the small ratio of $\mathrm{R}_{\text {ring }}$ and $\mathrm{R}_{\text {disk }}$. The large diffuse emission can be accounted for as the first expanding ring sweeps up the precollisional disk. The thickness ( $3.7 \pm 0.3 \mathrm{kpc}$; Methods) of the ring, the size ratio of the first to the second ring $\left(\sim 2.2\right.$, taking $R_{80}$ in the restframe optical as the radius of the first ring), SFR, age, and metallicity are broadly consistent with the analytical model predictions of successive rings 7

The diffuse light induced by the expanding ring is difficult to observe in local CRGs because of the low surface density of the redistributed stars ${ }^{25}$. R5519's extended disk has a rest-frame B-band (HST F160W) surface brightness of $\sim 20 \mathrm{AB}$ mag $\operatorname{arcsec}^{-2}$. Such a bright outer disk has yet to be seen in local CRGs (Supplementary Fig.14). The diffuse emission outside of R5519's ring ( $\gtrsim 6.5 \mathrm{kpc}$ ) contains $\gtrsim 50 \%$ of the total light in the rest-frame B band, whereas for local CRGs, most of the B-band luminosity is on and within the ring (Supplementary Fig.14). Without an intrinsic luminosity evolution with redshift, local CRGs' rings and extended disks would be undetectable at $z \approx 2$ with current observations (see Supplementary Information). However, if high-redshift CRGs follow the "main-sequence" relation at $z \approx 2$ as R5519 does, then they would be bright enough to be detected in the 3D-HST WFC3 images (Supplementary Fig.14).

An alternative scenario to explain the large diffuse light outside of the ring is through satellite perturbations. Recent CRGs in the Evolution and Assembly of GaLaxies and their Environments (EAGLE) simulation $\sqrt{13}$ show that interaction with multiple satellites at $z>2$ can cause $>50 \%$ of the stellar particles of the CRG host to be tidally perturbed outside of the ring $\sim 120$ Myr after the collision (Supplementary Figs.15-16). Similar to the EAGLE ring, the diffuse stellar light of R5519 could be tidally induced by small satellites or represents an ongoing accretion of small satellites. In this scenario, the ring can be either the first or a successive ring.

Both our observation, and the EAGLE simulations, imply that the volume number density of massive $\left(\log \left(\mathrm{M}_{*} / \mathrm{M}_{\odot}\right) \gtrsim 10.0\right)$ CRGs at $z \approx 2$ is as small as $z \sim 0$ (Supplementary Information). This seems contrary to previous predictions that CRGs are more common at high redshif $10-12$. Using a scaling relation of $(1+z)^{4.5}$ from a previous study 10 , CRGs are expected to be $\sim 140$ times more common at $z \approx 2$. Considering only the massive CRG hosts, the expected number density at $z \approx 2$ is still $\gtrsim 10 \times$ larger than at $z \sim 0$ (Methods). We speculate that a combined effect of a rising merger rate, a decreased fraction of large spiral disks, and the lack of local-like galaxy groups at high redshift could cause the slow CRG number density change $\frac{\sqrt{13}}{10}$ in the past $11 \mathrm{Gyr}$ (Supplementary Information). If R5519 is a density wave ring similar to local CRGs, it is an unequivocal sign of the existence of a thin disk in the young universe, critical for understanding the onset of spiral galaxies $20,21,30$.

\section{References}

1. Madore, B. F., Nelson, E. \& Petrillo, K. Atlas and Catalog of Collisional Ring Galaxies. Astrophys. J. Supp. 181, 572-604 (2009).

2. Lynds, R. \& Toomrel, A. On the interpretation of ring galaxies: the binary ring system II Hz 4. Astrophys. J. 209, 382-388 (1976).

3. Struck-Marcell, C. \& Higdon, J. L. Hydrodynamic models of the Cartwheel ring galaxy. Astrophys. J. 411, 108-124 (1993).

4. Appleton, P. N., Struck-Marcell, C. Collisional Ring Galaxies. FCPh 16, 111-220 (1996).

5. Higdon, J. L. Wheels of Fire. I. Massive Star Formation in the Cartwheel Ring Galaxy. Astrophys. J. 455, 524 (1995).

6. Gerber, R. A., Lamb, S. A. \& Balsara, D. S. A stellar and gas dynamical numerical model of ring galaxies. Mon. Not. R. Astron. Soc. 278, 345-366 (1996).

7. Struck, C. Applying the analytic theory of colliding ring galaxies. Mon. Not. R. Astron. Soc. 403, 1516-1530 (2010).

8. Mapelli, M. \& Mayer, L. Ring galaxies from off-centre collisions. Mon. Not. R. Astron. Soc. 420, 1158-1166 (2012). 
9. Higdon, J. L., Higdon, S. J. U., Martín Ruiz, S. \& Rand, R. J. Molecular Gas and Star Formation in the Cartwheel. Astrophys. J. 814, L1 (2015).

10. Lavery, R. J., Remijan, A., Charmandaris, V., Hayes, R. D. \& Ring, A. A. Probing the Evolution of the Galaxy Interaction/Merger Rate Using Collisional Ring Galaxies. Astrophys. J. 612, 679-689 (2004).

11. Elmegreen, D. M. \& Elmegreen, B. G. Rings and Bent Chain Galaxies in the GEMS and GOODS Fields. Astrophys. J. 651, 676-687 (2006).

12. D'Onghia, E., Mapelli, M. \& Moore, B. Merger and ring galaxy formation rates at $z<2$. Mon. Not. R. Astron. Soc. 389, 1275-1283 (2008).

13. Elagali, A. et al. Ring galaxies in the EAGLE hydrodynamical simulations. Mon. Not. R. Astron. Soc. 481, 2951-2969 (2018).

14. Genzel, R. et al. The SINS/zC-SINF Survey of $z \approx 2$ Galaxy Kinematics: Evidence for Gravitational Quenching. Astrophys. J. 785, 75 (2014).

15. Buta, R. J. \& Combes, F. Galactic Rings. Fund. Cosmic Phys. 17, 95-281 (1996).

16. Comeron, S. et al. ARRAKIS: atlas of resonance rings as known in the $\mathrm{S}^{4} \mathrm{G}$. Astron. Astrophys. 562, 121 (2014).

17. Sheth, K. et al. Hot Disks and Delayed Bar Formation. Astrophys. J. 758, 136 (2012).

18. Kraljic, F., Bournaud, F. \& Martig, M. The Two-phase Formation History of Spiral Galaxies Traced by the Cosmic Evolution of the Bar Fraction. Astrophys. J. 757, 60 (2012).

19. Cen, R. Evolution of Cold Streams and the Emergence of the Hubble Sequence. Astrophys. J. 789, L21 (2014).

20. Elmegreen, D. M. \& Elmegreen, B. G. The Onset of Spiral Structure in the Universe. Astrophys. J. 781, 11 (2014).

21. Vincenzo, F., Kobayashi, C. \& Yuan, T. Zoom-in cosmological hydrodynamical simulation of a star-forming barred, spiral galaxy at redshift $z=2$. Mon. Not. R. Astron. Soc. 488, 4674-4689 (2019).

22. Straatman, C. M. et al. The FourStar Galaxy Evolution Survey (ZFOURGE): Ultraviolet to Far-infrared Catalogs, Medium-bandwidth Photometric Redshifts with Improved Accuracy, Stellar Masses, and Confirmation of Quiescent Galaxies to $z \sim 3.5$. Astrophys. J. 830, 51 (2016).

23. Momcheva, I. G. et al. The 3D-HST Survey: Hubble Space Telescope WFC3/G141 Grism Spectra, Redshifts, and Emission Line Measurements for $\sim 100,000$ Galaxies. Astrophys. J. Supp. 225, 27 (2016).

24. Cowley, M. J. et al. ZFOURGE catalogue of AGN candidates: an enhancement of $160-\mu \mathrm{m}$-derived star formation rates in active galaxies to $z=3.2$. Mon. Not. R. Astron. Soc. 457, 629-641 (2016).

25. Romano, R., Mayya, Y. D. \& Vorobyov, E. I. Stellar Disks of Collisional Ring Galaxies. I. New Multiband Images, Radial Intensity and Color Profiles, and Confrontation with N-Body Simulations. Astron. J. 136, 12591289 (2008).

26. Tacconi, L. J. et al. High molecular gas fractions in normal massive starforming galaxies in the young Universe. Nature 463, 781-784 (2010).

27. Fogarty, L. et al. SWIFT observations of the Arp 147 ring galaxy system. Mon. Not. R. Astron. Soc. 417, 853-844 (2011).

28. Pearson, W. J. et al. Main sequence of star forming galaxies beyond the Herschel confusion limit. Astron. Astrophys. 615, A146 (2018).

29. van der Wel , A. et al. 3D-HST+CANDELS: The Evolution of the Galaxy Size-Mass Distribution since $z=3$. Astrophys. J. 788, 28 (2014).

30. Yuan, T.-T. et al. The Most Ancient Spiral Galaxy: A 2.6-Gyr-old Disk with a Tranquil Velocity Field. Astrophys. J. 850, 61 (2017).

Author Information Correspondence and requests for materials should be addressed to T.Y. (tiantianyuan@swin.edu.au).

Acknowledgements This research was supported by the Australian Research Council Centre of Excellence for All Sky Astrophysics in 3 Dimensions (ASTRO 3D), through project number CE170100013.

Author Contributions T.Y. wrote the manuscript and had the overall lead of the project. A.E. carried out the EAGLE simulation analysis and contributed to the writing of the simulation results. I.L.,G.K. and C.L. contributed significantly to the overall science interpretation, data analysis and making of the figures. L.A.,J.C., K-V.T., K.G. contributed significantly to the photometric and kinematic data analysis. All other coauthors contributed by their varied contributions to the science interpretation, data analysis and assistance with Keck observations. All co-authors contribute to the commenting on this manuscript as part of an internal review process.

Competing interests statement The authors declare no competing interests. 

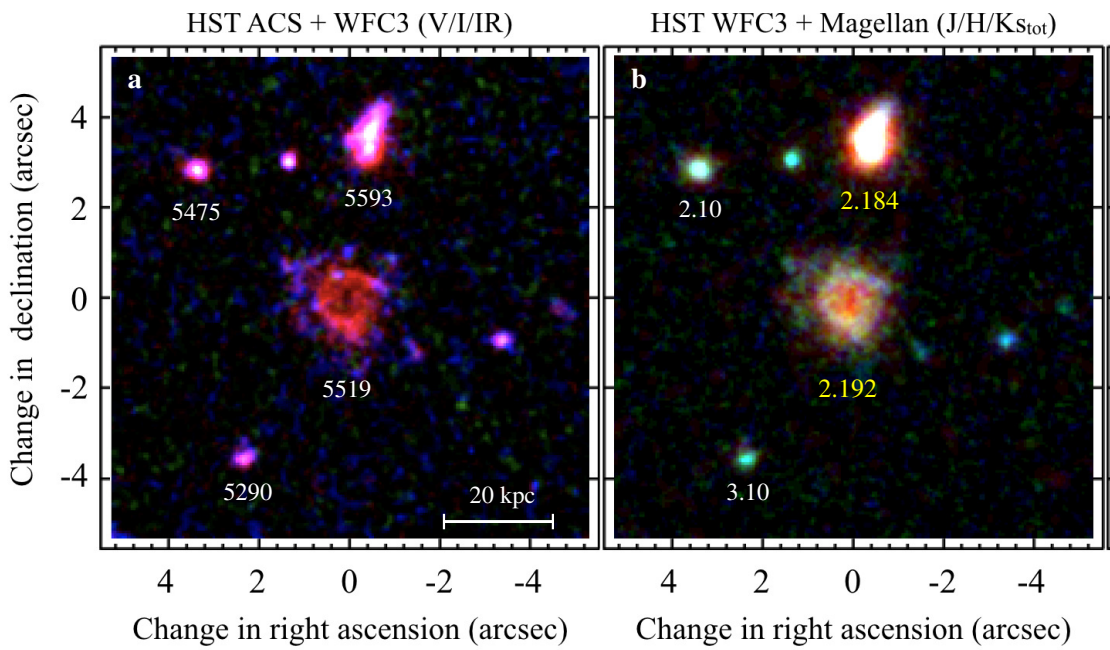

Rest-Frame (FUV/NUV/Optical)

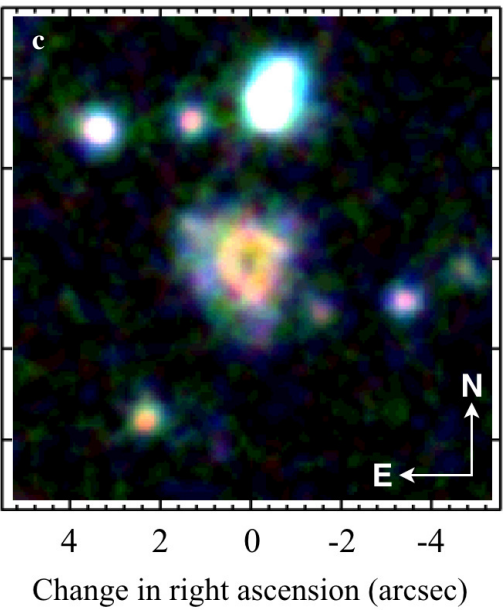

Figure 1 - Multi-wavelength views of $\mathbf{R 5 5 1 9}$ and and its neighbouring environment. a, a three-colour image combining HST V-band/ACS F606W (blue), I-band/ACS F814W (green), and IR-band/WFC3 F125W+F140W+F160W (red) images. Panel a shows the highest spatial resolution (full width at half maximum $\sim 1.7-2.2 \mathrm{kpc}$ ) view of R5519. The numbers under each object denote the ZFOURGE catalogue ID. The contrast of the image is tuned to highlight the double nuclei and tail-like structure of the companion galaxy G5593. b, Blue/Green/Red (HST F125W/F160W/Magellan Ks tot) colour image. The Ks tot image is a super deep K-band detection image in ZFOURGE (Methods). This image highlights the resolved ring structure on top of the longest-wavelength image from the ground-based ZFOURGE Ks tot band (rest-frame R). The ZFOURGE photometric redshifts are labelled in white, with confirmed spectroscopic redshifts in yellow. c, Blue/Green/Red (combined rest-frame FUV/NUV/optical) colour image. The images are generated by stacking HST and ZFOURGE catalogue images that correspond to rest-frame FUV, NUV, and optical wavelengths (see Supplementary Information). The pixel scale for image a is 0." 06 as sourced from the 3D-HST survey. For b-c, a pixel scale of $0 . \prime 15$ is used to match the ground-based images 22 . A logarithmic stretch is used for all images in this work.

\begin{tabular}{|c|c|c|c|}
\hline Objects & R5519 & G5593 & G5475 \\
\hline Right ascension (J2000) & $10^{h} 00^{\min } 43.847^{s}$ & $10^{h} 00^{\min } 43.815^{s}$ & $10^{h} 00^{\min } 44.081^{s}$ \\
\hline Declination (J2000) & $+214^{\prime} 05.476^{\prime \prime}$ & $+214^{\prime} 09.167^{\prime \prime}$ & $+214^{\prime} 08.469^{\prime \prime}$ \\
\hline Redshift $z$ & $2.1927_{-0.0001}^{+0.0001}$ & $2.184_{-0.006}^{+0.005}$ & $2.10_{-0.07}^{+0.07}$ \\
\hline Stellar mass $\left(\log _{10}\left(\mathrm{M}_{*} / \mathrm{M}_{\odot}\right)\right)$ & $10.78_{-0.03}^{+0.03}$ & $10.40_{-0.04}^{+0.04}$ & $9.55_{-0.04}^{+0.03}$ \\
\hline $\mathrm{SFR}_{\mathrm{IR}+\mathrm{UV}}\left(\mathrm{M}_{\odot} \mathrm{yr}^{-1}\right)$ & $80_{-0.2}^{+0.2}$ & $123_{-2}^{+2}$ & 0 \\
\hline $\mathrm{SFR}_{\mathrm{H} \alpha}\left(\mathrm{M}_{\odot} \mathrm{yr}^{-1}\right)$ & $38-48$ & - & - \\
\hline \multicolumn{4}{|l|}{$\Sigma_{\mathrm{SFR}}\left(\mathrm{M}_{\odot} \mathrm{yr}^{-1} \mathrm{kpc}^{-2}\right)$} \\
\hline$i\left(^{\circ}\right)$ & $29_{-5}^{+5}$ & - & - \\
\hline$P A\left(^{\circ}\right)$ & $28_{-10}^{+10}$ & - & - \\
\hline Ring average radius $(\mathrm{kpc})$ & $5.1_{-0.4}^{+0.4}$ & - & - \\
\hline inner radius (kpc) & $2.7_{-0.2}^{+0.2}$ & - & - \\
\hline outer radius $(\mathrm{kpc})$ & $6.5_{-0.2}^{+0.2}$ & - & - \\
\hline ring thickness (kpc) & $3.7_{-0.3}^{+0.3}$ & - & - \\
\hline $\mathrm{R}_{50}(\mathrm{kpc})$ & $7.6_{-0.2}^{+0.2}$ & $3.3_{-0.1}^{+0.1}$ & $1.5_{-0.2}^{+0.2}$ \\
\hline $\mathrm{R}_{80}(\mathrm{kpc})$ & $11.8_{-0.3}^{+0.3}$ & $4.9_{-0.2}^{+0.2}$ & $2.5_{-0.2}^{+0.2}$ \\
\hline Rotational velocity $\mathrm{V}_{\text {rot }}\left(\mathrm{km} \mathrm{s}^{-1}\right)$ & $90_{-75}^{+75}$ & - & - \\
\hline Radial (expansion) velocity $\mathrm{V}_{\text {rad }}\left(\mathrm{km} \mathrm{s}^{-1}\right)$ & $226_{-90}^{+90}$ & - & - \\
\hline
\end{tabular}

Table 1 - Physical properties of R5519 and its neighbours. The $z$ values for R5519, G5593, and G5475 are MOSFIRE $z_{\mathrm{s}}$, HST $z_{\text {grism }}$, and ZFOURGE $z_{\mathrm{p}}$, respectively. For all sources, the stellar masses are calculated based on the spectral energy distribution from the ZFOURGE photometry and the SFR IR+UV are derived on the basis of the combined rest-frame IR and UV luminosities. The range of SFR $\mathrm{H}_{\alpha}$ values reflects different assumptions about attenuation laws. $\Sigma_{\mathrm{SFR}}$ is estimated by dividing the total SFR by an assumed total SFR area, with the range of values reflecting different assumptions (Methods). PA and $i$ are measured on the basis of the ellipse fit to the combined HST F125W+F140W+F160W image. The inner and outer radii and thickness of the ring are derived from a double ellipse fit, whereas the average radius is based on a single ellipse fit (Methods,Supplementary Fig.1). $\mathrm{R}_{50}$ and $\mathrm{R}_{80}$ are the radii where $50 \%$ and $80 \%$ of the total luminosity are enclosed in the combined HST F125+F140W+F160W band, respectively. The rotational and radial velocities are derived at the 5 kpc ring radius using a tilted rotating and expanding circular ring model (Methods). 


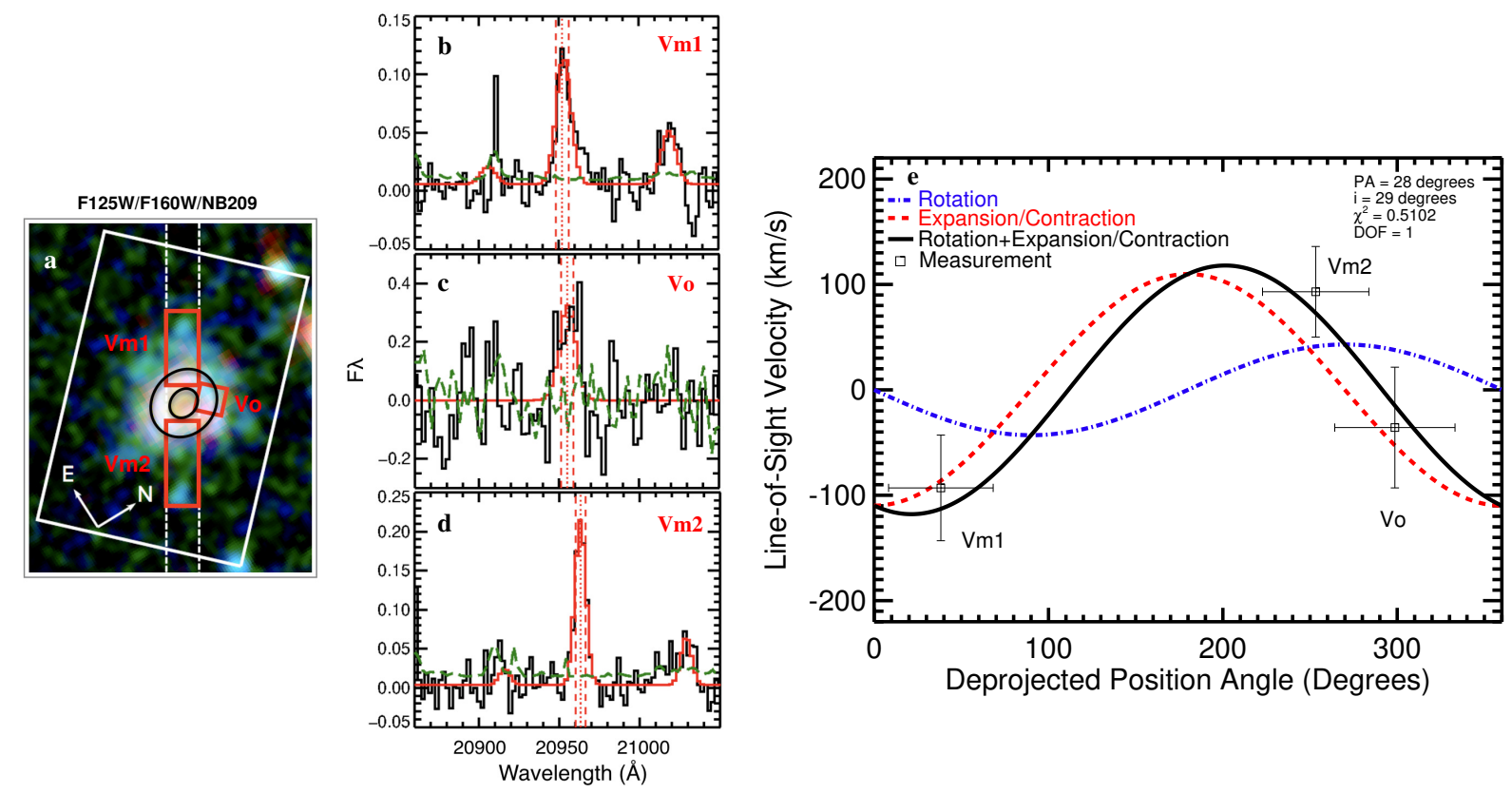

Figure 2 - A joint analysis of the MOSFIRE and OSIRIS ring kinematics. a, Spatial alignment of the OSIRIS (white box; field-of-view 4 ." $5 \times 6 . " 4$ ) and MOSFIRE (white dashed box; slit width 0." 8 ) observations on the Blue/Green/Red three-colour image (HST F125W/F160W/ ZFOURGE NB209 H $\alpha$ narrow band). The black circles show the best-fit double ellipse to the ring structure. The red boxes mark the three position angles where the line-of-sight velocities (MOSFIRE: Vm1, Vm2; OSIRIS: Vo) are measured (see also Supplementary Figs.5-6). b-d, The observed $\mathrm{H} \alpha$ spectra (black), best-fit (red) and $1 \sigma$ noise (green) for Vm1, Vm2 and Vo. F $\lambda$ is the observed flux of the spectra. The red vertical dotted and dashed lines show the the determined velocity centre and its uncertainty range, respectively. The uncertainty range is a conservative measure using the $1 \sigma$ width of a Gaussian line profile (Methods). The flux unit for $\mathbf{b}, \mathbf{d}$ is $10^{-17} \mathrm{ergs} / \mathrm{s} / \mathrm{cm}^{2} / \AA$, and for $\mathbf{c}$, the flux unit is $10^{-20} \mathrm{ergs} / \mathrm{s} / \mathrm{cm}^{2} / \AA$. e, Fitting an expanding/contracting tilted circular ring model to the line-of-sight velocity versus deprojected position angle diagram. The vertical errors of $\mathrm{Vm} 1, \mathrm{Vm} 2$, and $\mathrm{Vo}$ are defined by the uncertainty range of the line centre in b-d; the horizontal error is defined by the alignment uncertainty in a. The model uses a fixed inclination $i=29^{\circ}$ and a kinematic major axis of $P A=28^{\circ}$. The black curve is the best-fit model with contributions from both rotation (blue) and expansion/contraction (red). The expansion/contraction component is detected for a varied range of $P A=0-45^{\circ}$ and $i=20-45^{\circ}$ (Methods, Supplementary Figs.7-9). 


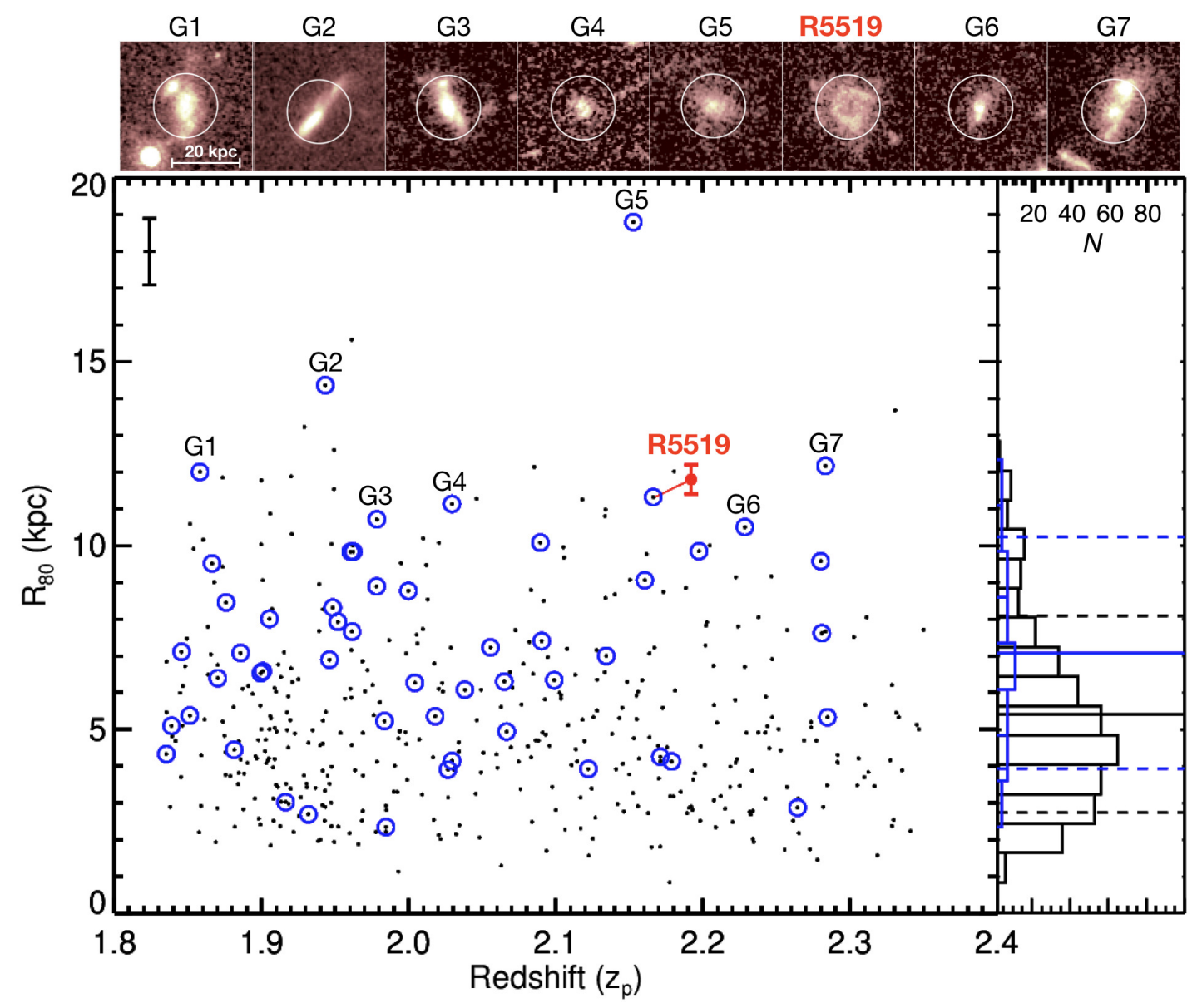

Figure 3 - Comparing the size of $\mathbf{R 5 5 1 9}$ with the size distribution of late-type galaxies at $z \approx 2$. The $z_{\mathrm{p}}$ and $\mathrm{R}_{80}$ values are from the $3 \mathrm{D}-\mathrm{HST}$ survey and its mass-size catalogue in the COSMOS field (Methods). The typical error bar for $\mathrm{R}_{80}$, defined as an average error propagated through the $1 \sigma$ model error of the effective radius in 3D-HST, is shown in the top left corner. The black dots show all galaxies with $\log \left(\mathrm{M}_{*} / \mathrm{M}_{\odot}\right) \geq 9.5$. The blue circles highlight the most massive objects, defined as $\log \left(\mathbf{M}_{*} / \mathbf{M}_{\odot}\right) \geq 10.6$. The histograms (right) show the size distribution for all (black) and the most massive (blue) galaxies. The horizontal solid and dashed lines mark the mean and the $1 \sigma$ scatter of the size distributions. Including R5519, there are eight objects (labelled G1-G8) that have unusually large sizes (defined as $1 \sigma$ above the mean size of the most massive galaxies). The F160W band morphologies for these eight objects are shown as postage-stamp images at the top. The red filled circle shows $z_{\mathrm{s}}$ and our non-parametric measurement of $\mathrm{R}_{80}$ for $\mathrm{R} 5519$. We note that the inferred $\mathrm{R}_{80}$ for the 3D-HST sample is based on an empirical relation for a large sample of galaxies; individual galaxies may deviate from this relation due to scatter (Methods). 


\section{METHODS}

Throughout we adopt a $\Lambda$ cold dark matter cosmology, where $\Lambda$ is the cosmological constant, with $\Omega_{M}=0.307, \Omega_{\Lambda}=0.693$, and $\mathrm{H}_{0}=67.7 \mathrm{~km} \mathrm{~s}^{-1} \mathrm{Mpc}^{-1}$, consistent with the Planck measurements ${ }^{31}$ and the cosmological parameters used in EAGLE simulations 13 . At the redshift of $z=2.19$, the look-back time is $10.8 \mathrm{Gyr}$ and one arcsecond corresponds to a physical scale of $8.49 \mathrm{kpc}$. All magnitudes are in $\mathrm{AB}$ units ${ }^{32}$, unless otherwise specified.

\section{Size of the Ring and the Extended Diffuse Emission}

1.1 Size of the ring We use the original 3D-HST CANDELS version of the F125W, F140W and F160W images 33,34 to quantify the size of the ring structure. We fit both a single ellipse and a double ellipse of a constant width to the imaging data using a $\chi^{2}$ minimisation procedure. In both approaches, we divide the ring azimuthally into $\mathrm{N}=180$ intervals and use the average ful width at half maximum (FWHM) of the surface brightness (SB) along each azimuthal angle interval to determine the inner and outer edges of the ring The baseline of the FWHM is chosen as the average SB in the central pixels of the ring (red cross in Supplementary Fig.1). We smooth the images by 3 pixels $\left(0 .{ }^{\prime \prime} 18\right)$ to enhance the signal-to-noise ratio (SNR).

In the single-ellipse approach, we use data points on the outer edge of the ring as the input and weight each azimuthal angle with the median SB within each azimuthal interval. The free parameters of a single ellipse model are: the centre $(x c, y c)$, major axis radius $(a)$, axis ratio $(b / a$ or inclination angle $i$ ) and position angle (PA). In the double ellipse approach, we use the inner and outer edges of the ring as double constraints and weigh each azimuthal angle with the median SB within each azimuthal interval. The additional free parameter in the double ellipse model is the width $(\Delta \mathrm{R})$ of the ring. We find that both approaches provide reasonably good fits to the data (Supplementary Table 1). We carry out the fit on both the single bands (F125W, F160W) and the combined band (F125W+F140W+F160W). The results are consistent within $1 \sigma$ of the statistical errors. The best-fit ellipses and parameters are shown in Supplementary Fig.1 and Supplementary Table 1.

1.2 Size of the diffuse stellar light We quantify the size of the diffuse stellar light in a model-independent way by measuring the accumulated luminosity within a circular aperture of an increasing radius (Supplementary Fig.12). We calculate $\mathrm{R}_{50}, \mathrm{R}_{80}$, and $\mathrm{R}_{95}$ where $50 \%, 80 \%$ and $95 \%$ of the total luminosity is enclosed. The choice of the three radii is to facilitate comparison with other samples of galaxies: $\mathrm{R}_{50}$ is comparable to the effective radius $\mathrm{R}_{e}$ of a Sersic profile; $\mathrm{R}_{80}$ is a recently popularised parameter to study the size evolution of galaxies with redshift 35 ; $R_{95}$ describes the outer edge of the galaxy. For a Sersic profile of $n=0.5$ and $n=1.0, \mathrm{R}_{95}$ corresponds to a radius of $\sim 2.1 \mathrm{R}_{e}$ and $\sim 2.9 \mathrm{R}_{e}$, respectively 36 .

The diffuse stellar light of R5519 is present in multiple wavebands (Supplementary Fig.2). To test the dependence of the measured size on wavelength, image depth, and the point spread function (PSF), we carry out the measurement on the deep ZFOURGE Ks $s_{\text {tot }}$ image and the high-spatial resolution HST F125+F140W+F160W image; we then repeat the measurements on the PSF matched ZFOURGE image, including a stacked rest-frame FUV image. The PSF is best characterised by a Moffat profile of two parameters: FWHM and $\beta$, where $\beta$ describes the overall shape of the PSF. The PSF matched images are carefully generated by the ZFOURGE team using a Moffat profile with FWHM of $0 . \prime 9$ and $\beta=0.9$ [ref. ${ }^{22}$ ]. Our stacked rest-frame FUV image is produced from the PSF-matched ground-based UBGV images (see Supplementary Information).

We summarise the derived $\mathrm{R}_{50}, \mathrm{R}_{80}$, and $\mathrm{R}_{95}$ in Supplementary Table 4. The error bars are derived by perturbing the measurements within $1 \sigma$ of the sky background. The PSF-matched images yield on average a larger size of $1.4 \pm 0.6 \mathrm{kpc}$ at all wavelengths. For images of similar depth and PSF the bluest wavelength yields the largest size, e.g., the rest-frame FUV size is $\sim 1 \mathrm{kpc}$ larger than the rest-frame B band. Note that the diffuse stellar light distribution of R5519 is not circularly symmetric, our $R_{50}, R_{80}$, and $R_{95}$ can be considered as circularly averaged values. These circularly averaged values are consistent with the size measurement from the surface brightness distribution along the major axis of the ring ellipse below.
1.3 Surface brightness profile in 1D along the major axis We measure the 1D surface brightness distribution $\mathrm{SB}(\mathrm{R})$ by averaging three slices along the major axis of the ring ellipse in the deep HST F160W image (Supplementary Fig.13). The three slices are chosen as the best-fit major axis and its $1 \sigma$ upper and lower limits (red solid and dashed lines in Supplementary Fig.13). Each datapoint along the slice is an average of 4 pixels $(0.24)$ in width, i.e., about one image resolution element $\left(00^{\prime \prime} 26\right)$. We stop the measurements when the data are indistinguishable from the $1 \sigma$ fluctuation of the background noise. A total size of the galaxy is defined by the boundary where the $1 \mathrm{D} \mathrm{SB}$ drops to the $1 \sigma$ background noise level. We estimate R5519's total size to be $\mathrm{R}_{t o t}=15 \pm 1 \mathrm{kpc}$ in radius, with the error bar indicating the uncertainty in identifying the boundary that is consistent with the noise. The $\mathrm{R}_{t o t}$ agrees with our measured $\mathrm{R}_{95}=15.6 \pm 0.6 \mathrm{kpc}$ using the circular aperture on the combined F125W+F140W+F160W image (Supplementary Table 4$)$. We measure the ring's inner and outer radius $\left(\mathrm{R}_{\text {in }}\right.$ and $\mathrm{R}_{\text {out }}$ ) based on the FWHM of the ring feature. We use the SB in the centre of the ring as the baseline of the FWHM. We find $\mathrm{R}_{\text {in }}=2.1 \mathrm{kpc}$ and $\mathrm{R}_{\text {out }}=6.7$ kpc, broadly consistent with the 2D double ellipse fit (Supplementary Table 1).

The method we use to derive $\mathrm{R}_{t o t}$ and $\mathrm{R}_{95}$ may not be practical for local CRGs such as the Cartwheel galaxy, where the ring dominates the luminosity in the outer disk (e.g., Supplementary Fig.14). The method we use for $\mathrm{R}_{t o t}$ is very similar to the commonly used $\mathrm{R}_{B 25}$ for local $\mathrm{CRG} s \frac{\sqrt{25}}{}$. $\mathrm{R}_{B 25}$ refers to the radius where the SB drops to a standard level of surface brightness of $25.00 \mathrm{mag} \operatorname{arcsec}^{-2}$ in the B band for angular dimensions 37 . Instead of using a fixed SB, we use the $1 \sigma$ sky background that is more suitable for high-redshift observations.

The average SB of the diffuse disk estimated from the average light between $\mathrm{R}_{\text {out }}$ and $\mathrm{R}_{95}$ is $0.42 \mu \mathrm{Jy} \operatorname{arcsec}^{-2}$ in F160W. Assuming a cosmological SB dimming form of $(1+z)^{-4}$, the average SB of R5519's diffuse disk observed at $z \sim 0$ would be 19.8 mag $\operatorname{arcsec}^{-2}$ in the B band. This is almost four magnitudes brighter than the SB of the brightest outer disk of nearby CRG 5 . Using the average SB of the diffuse disk as the baseline of the FWHM yields a $\sim 0.5 \mathrm{kpc}$ increase/decrease in the size of the inner/outer radius. The average $\mathrm{SB}$ of the ring as calculated between $\mathrm{R}_{\text {in }}$ and $\mathrm{R}_{\text {out }}$ is 1.04 $\mu \mathrm{Jy} \operatorname{arcsec}^{-2}$. The peak SB inside the ring is $1.15 \mu \mathrm{Jy} \operatorname{arcsec}^{-2}$. Therefore the relative SB between the ring and the outer disk is 0.6-0.7 $\mu \mathrm{Jy} \operatorname{arcsec}^{-2}$. 1.4 Comparing with 3D-HST $z \approx 2$ late-type galaxies To put the size of R5519 in context with other $z \approx 2$ galaxies, we compare its $\mathrm{R}_{80}$ with 3DHST galaxies measured on the same F160W images $\sqrt{29}$ in Fig.3. The sizes of 3D-HST galaxies have been modelled by Sersic profiles through several well-established studies $29[35,38$. We use the mass-size catalogue data from the 3D-HST survey ${ }^{29}$ and apply the same conversion between Sersic index $n$, effective radius $\mathrm{R}_{e}$ and $\mathrm{R}_{80}$ as previous studies 29,35$] 38$. In order to minimise systematic errors of $\mathrm{R}_{80}$, we only include galaxies with flux $\mathrm{SNR}>20$ on the F160W image and have good Sersic model fits (SNR $>5$ for both $\mathrm{R}_{e}$ and $n$ ). We select data with 3D-HST photometric redshifts $(z p)$ of $1.8<z<2.4$ and $\log \left(\mathrm{M}_{*} / \mathrm{M}_{\odot}\right)>9.5$, to match our ZFOURGE target selection criteria. Only late-type $(n<2.5)$ galaxies are used. We then cross-correlate the 3DHST $z p$ with the high-precision $(\sim 2 \%)$ ZFOURGE photometric redshifts ${ }^{\sqrt{22}}$ and exclude targets with $z p<1.5$ or $z p>2.5$. We also exclude targets that have inconsistent stellar masses $\left(\Delta \log \left(\mathrm{M}_{*} / \mathrm{M}_{\odot}\right)>0.5\right)$ from these two catalogues. Targets without ZFOURGE $z p$ or $\mathrm{M}_{*}$ remain in the sample. A total of $N=440$ objects satisfy these selection criteria.

The empirical conversion between $\mathrm{R}_{80}, \mathrm{R}_{e}$, and $n$ is based on large samples. The conversion is not guaranteed for individual galaxies. For example, the $\mathrm{R}_{80}$ for G4 and G5 in Fig.3 is probably inaccurate and reflects the scatter in the empirical conversion. R5519 was included in the Sersic profile modelling of previous 3D-HST studies 29 . The inferred $\mathrm{R}_{80}$ of R5519 from the 3D-HST catelogue ${ }^{29}$ Sersic $\mathrm{R}_{e}(7.6 \mathrm{kpc})$ and $n(0.42)$ is $11.2 \mathrm{kpc}$, in broad agreement with our non-parametric measurement $(11.8 \pm 0.3 \mathrm{kpc})$. Our model-independent $\mathrm{R}_{50}$ for R5519 is also consistent with the Sersic modelbased $\mathrm{R}_{e}$ from the 3D-HST catalogue ${ }^{29}$ within the measurement errors. Both the $\mathrm{R}_{80}$ and $\mathrm{R}_{50}$ of $\mathrm{R} 5519$ are $1.5 \sigma$ above the scatter of the late-type galaxies at $z \approx 2$ of similar or higher stellar masses $\left(\log \left(\mathrm{M}_{*} / \mathrm{M}_{\odot}\right) \geq 10.6\right)$. We conclude that $\mathrm{R} 5519$ is an unusually large galaxy at $z \approx 2$ regardless of the methods we use to quantify its total size.

The large size of R5519 can be further appreciated when compared with 
the Milky Way. The scale length $\left(\mathrm{R}_{s}\right)$ of the Milky Way's stellar disk is in the range of 2-3 kpc based on a large body of literature 39.41 . Using $R_{50}=1.68$ $\mathrm{R}_{s} \sqrt[36,42]{ }$ and $\mathrm{R}_{80} \sim 3.2 \mathrm{R}_{s}^{\sqrt[43]{3}}$, our Milky Way has $\mathrm{R}_{50}=3.4-5.0 \mathrm{kpc}$ and $\mathrm{R}_{80}=6.4-$ $9.6 \mathrm{kpc}$ in its stellar light. According to our best size estimation from HST WFC3 images (Table 1), the $\mathrm{R}_{50}$ of R5519 is 1.5-2.2 times larger and $\mathrm{R}_{80}$ is 1.2-1.8 times larger than the Milky Way.

\section{A Joint Analysis of MOSFIRE and OSIRIS H $\alpha$ Kinemat- ics}

The line-of-sight velocity ( $\left.V_{\mathrm{LOS}}\right)$ as a function of de-projected position angle $(\psi)$ at a fixed radius on the ring is one of the commonly used methods to derive the expansion/contraction and rotational velocities of CRGs Following similar analysis of local CRGs $\sqrt{27,44 / 46}$, we use equations below to describe the relation between $V_{\mathrm{LOS}}$ and $\psi$ in a tilted rotating and expanding/contracting circular ring model. An illustration of the geometry and definition of parameters is presented in Supplementary Fig.5:

$$
\begin{aligned}
\mathrm{V}_{\mathrm{LOS}, \psi}= & \left(\mathrm{V}_{\text {sys }}+\left(\mathrm{V}_{\text {rad }} \cos (\psi)-\mathrm{V}_{\text {rot }} \sin (\psi)\right)\right) \sin (\mathrm{i}), \\
& \tan (\psi)=\tan \left(\psi_{\mathrm{o}}\right) \cos (\mathrm{i}) ; \mathrm{i} \neq 90^{\circ} .
\end{aligned}
$$

$V_{\mathrm{LOS}, \psi}$ is the line-of-sight velocity measured at $\psi$ on the ring and $V_{\text {sys }}$ is the systematic velocity. $V_{\mathrm{LOS}}$ is calculated with respect to the kinematic centre of the galaxy. We take the cosmological expansion out by using the systematic redshift measured on the $\mathrm{H} \alpha$ centroid of the total aperture MOSFIRE spectrum $\left(z_{s, t o t a l}\right)$, hence we have $\mathrm{V}_{\text {sys }} \approx 0$ if $z_{s, \text { total }}$ is close to the systematic redshift at the kinematic centre. A positive sign of $V_{\text {LOS }}$ means redshift whereas a negative sign means blueshift. $\mathrm{V}_{\text {rad }}$ is the expansion/contraction velocity at the fixed radius of the ring. $\mathrm{V}_{\text {rot }}$ is the rotation velocity. The angle $\psi$ is measured counterclockwise from the kinematic major axis. The relation between the observed position angle $\left(\psi_{o}\right)$ and the deprojected $\psi$ is a simple function of the inclination angle $i$, where $i=0$ means viewing the disk of the ring face-on and $i=90$ means edge-on. The corrections between $\psi_{o}$ and $\psi$ are small for face-on disks, whereas the equations are invalid for edge-on disks (e.g., Supplementary Fig.5).

Note that in Eq. 11 the signs of $\mathrm{V}_{\text {rad }}$ and $\mathrm{V}_{\text {rot }}$ can be either positive or negative. In a fiducial case in Supplementary Fig.5, we define an east and a west side similar to the compass on a 2D image. In this case, the east side (with respect to the kinematic major axis) is the far side of the disk relative to the observer, a positive/negative sign of $\mathrm{V}_{\text {rad }}$ means expanding/contracting radially, and a positive/negative sign of $\mathrm{V}_{\text {rot }}$ means rotating counterclockwise/clockwise from the kinematic major axis. The signs of $\mathrm{V}_{\text {rad }}$ and $\mathrm{V}_{\text {rot }}$ are flipped if the east side is the near side of the ring. Without knowledge of the near and far side of the ring, the equations above provide only a magnitude of the radial velocity $\left(\mathrm{V}_{\text {rad }}\right)$ and rotational velocity $\left(\mathrm{V}_{\text {rot }}\right)$. In the nearby universe, the near and far side of the ring can be determined from an extinction-reddening asymmetry across the minor axis in a tilted galactic disk. This asymmetry is most evident when a galaxy has a prominent bulge: the bulge is viewed through the dust layer on the near side, while the dust is viewed through the bulge on the far side. However, this method is difficult to apply to high-redshift galaxies whose bulge and disk are in the early stages of formation.

The relation between $V_{\mathrm{LOS}}$ and $\psi$ at a fixed radius (R) is therefore a function of four parameters: the systematic velocity $\left(\mathrm{V}_{\text {sys }}\right)$, the circular rotational velocity $\left(V_{\text {rot }}\right)$, the expansion/contraction velocity $\left(V_{\text {rad }}\right)$ and the inclination angle $i$ (Supplementary Fig.5). One additional hidden parameter is the position angle $\left(\mathrm{PA}_{0}\right)$ of the kinematic major axis because $\psi$ is measured with respect to $\mathrm{PA}_{0}$. We do not have direct measurement of $\mathrm{PA}_{0}$ and make the assumption that $\mathrm{PA}_{0}$ is the position angle of the geometric major axis. We discuss the consequence of this assumption in the SI.

We can obtain three measurements on the $V_{\mathrm{LOS}}$ versus $\psi$ diagram by combining our MOSFIRE and OSIRIS $\mathrm{H} \alpha$ kinematics. The first two measurements are derived from the MOSFIRE slit spectrum and the third measurement comes from the OSIRIS observation. Our MOSFIRE $\mathrm{H} \alpha$ velocity is spatially resolved and a clear relative wavelength separation is seen in the $+y$ and $-y$ spatial direction of the spectrum (Supplementary Fig.6). We align the MOSFIRE $\mathrm{H} \alpha$ 2D spectra in its spatial y direction with the NB209 $\mathrm{H} \alpha$ narrow-band image. We do this by cross-correlating the spatial $\mathrm{H} \alpha$ line profiles of the MOSFIRE observation with a mock $\mathrm{H} \alpha$ spatial line profile derived from the $\mathrm{H} \alpha$ narrow-band image. In Supplementary Fig.6, we show the MOSFIRE spectrum aligned spatially with the NB209 image after correcting for the central offset; the error bar $\left(\sim 00^{\prime \prime} 2\right)$ of the alignment is estimated by the $1 \sigma$ scatter of 100 times refit to the convoluted NB209 image using a full range of seeing $\left(0{ }^{\prime \prime} 6-0\right.$. . 9 ) sizes experienced in our MOSFIRE observations. We then align the OSIRIS datacube with the NB209 $\mathrm{H} \alpha$ narrow band image using the corrected astrometry (see Supplementary Information). The maximum error of the OSIRIS to NB209 alignment is the spatial resolution of our adaptive optics (AO) observations ( 0 "' 3 ).

Our measurement is then carried out at the ring radius $(\mathrm{R}=5 \mathrm{kpc})$ for 3 positions on the ring. We assume that the best-fit ellipse PA is the kinematic position angle $\mathrm{PA}_{0}$ and fix the inclination angle at the best value of $29^{\circ}$ as derived from the ellipse fitting. The uncertainties in $\psi$ are taken as the edge of the MOSFIRE slit and the edge of the OSIRIS $\mathrm{H} \alpha$ detection box (Supplementary Fig.6). The line-of-sight velocities from the MOSFIRE spectra are based on the $\mathrm{H} \alpha$ emission line centroid of the 1D spectra extracted at the $5 \mathrm{kpc}$ location (Fig.3). Due to the seeing-limited nature of the MOSFIRE observation, the 1D spectra extracted at the $5 \mathrm{kpc}$ position $( \pm 1$ pixel $)$ on MOSFIRE is prone to the uncertainty of the PSF characterisation. We test the uncertainties of $\mathrm{Vm} 1$ and $\mathrm{Vm} 2$ by shifting the extracting centre of the $1 \mathrm{D}$ spectra within the range of the spatial alignment error $(\sim 0,2)$. Because of the strong correlation of the spatial position and the split of the blueand redshift of the 2D spectrum, we find that the Gaussian width of the line profiles provides a conservative estimate of the measurement uncertainty in $\mathrm{Vm} 1$ and $\mathrm{Vm} 2$. As long as the kinematic field is relatively face-on $\left(<45^{\circ}\right)$, the effect of beam-smearing in deriving the $\mathrm{H} \alpha$ line centroid is budgeted into the uncertainty from the line width. The low SNR of our OSIRIS spectrum prompts us to use the line width as an upper limit for the uncertainty in determining the line centroid. The uncertainties of the line-of-sight velocities along the MOSFIRE PA (Vm1, Vm2) and OSIRIS $\mathrm{H} \alpha(\mathrm{Vo})$ are therefore taken as the Gaussian width of the line profiles. We also note that whether the measurement is done on a $5 \mathrm{kpc}$ radius circle or the best-fit ellipse does not change the result.

We fitted our three data points with Eq. 1.2 using a $\chi^{2}$ minimisation procedure, weighted by the inverse square of the total error $\sigma_{x, y}=$ sqrt $\left(\sigma^{2}{ }_{\left(V_{\text {LOS }, \psi}\right)}+\sigma^{2}{ }_{(\psi)}\right)$. We keep $\mathrm{V}_{\text {sys }}$ at 0 , though allowing it as a free parameter to account for any offsets between $z_{\text {s,total }}$ and the redshift from the actual kinematic centre of the disk does not change our main conclusion. For our fixed $\mathrm{PA}_{0}=28^{\circ}$ and $i=29^{\circ}$, the best-fit expansion/contraction velocity is $\mathrm{V}_{\mathrm{rad}}=226 \pm 90 \mathrm{~km} / \mathrm{s}$ and $\mathrm{V}_{\text {rot }}=90 \pm 75 \mathrm{~km} / \mathrm{s}$ (Fig.3). The systematic errors of our kinematic measurement are discussed in the Supplementary Information. Nearby CRGs show a range of $V_{\text {rad }}(50-220 \mathrm{~km} / \mathrm{s})$ and $V_{\text {rot }}$ $(50-350 \mathrm{~km} / \mathrm{s})$. For most nearby CRGs, $\mathrm{V}_{\text {rot }}$ is larger than $\mathrm{V}_{\text {rad }} \sqrt[9.44]{\text {, though }}$ in some cases (e.g., Arp 147), $\mathrm{V}_{\text {rot }}$ can be a few times smaller than $\mathrm{V}_{\text {rad }}$.27.

\section{Colour, Stellar Mass and Age}

3.1 Global We estimate the total stellar mass and average age of the stellar population via spectral energy distribution (SED) fitting to ZFOURGE multi-band photometry. The total photometry is measured on PSF matched ZFOURGE images.

The publicly available catalogue of ZFOURGE provides stellar masses based on the SED-fitting code FAST ${ }^{[47}$ in combination with the photometric redshift from EAzY ${ }^{48}$. FAST determines the best-fit parameters of SED models through a $\chi^{2}$ minimisation procedure 49 . We use 36 passbands of ZFOURGE's total photometry based on the stellar population synthesis (SPS) model grids ${ }^{50}$, a Chabrier IMF ${ }^{51}$, a fixed solar metallicity $\left(1.0 \mathrm{Z}_{\odot}\right)$, an exponentially declining star formation history (SFH), and a Calzett ${ }^{[52}$ extinction law. The ZFOURGE FAST output catalogue 49 records a total stellar mass of $\log \left(\mathrm{M}_{*} / \mathrm{M}_{\odot}\right)=10.78 \pm 0.03$ for R5519, $10.40 \pm 0.04$ for G5593, and $9.89 \pm 0.09$ for G5475. We test the systematic uncertainties of $\mathbf{M}_{*}$ against different SED fitting packages and find a systematic error of $\sim 0.2$ dex (see in Supplementary Information).

To obtain a lower and upper limit for the stellar mass of R5519 we run FAST assuming two extreme SFH: a constant star-formation model (CSF) with emission lines and a $10 \mathrm{Myr}$ truncated burst with no star formation afterwards (Supplementary Fig.10). The CSF provides an upper limit to 
the stellar mass and age of R5519: $\log \left(\mathrm{M}_{*} / \mathrm{M}_{\odot}\right)<10.8$ and $\mathrm{t}_{\text {age }}<2 \mathrm{Gyr}$ The truncated star formation model provides a lower limit of $\log \left(\mathrm{M}_{*} / \mathrm{M}_{\odot}\right)$ $>10.5$ and $\mathrm{t}_{\text {age }}>50 \mathrm{Myr}$. These are the best constraints we can provide for R5519 from current SED analysis.

3.2 Spatially resolved photometry In ZFOURGE, a super deep K-band detection image $\left(\mathrm{Ks}_{\text {tot }}\right)$ was created by combining FourStar/Ks-band observations with pre-existing $\mathrm{K}$-band images 2 . The $\mathrm{Ks}_{\text {tot }}$ image reveals an offcentre region that we speculate could be the "nucleus" of the pre-collisional galaxy (Supplementary Fig.2). We measure the aperture photometry of this postulated "nucleus" region and compare it with regions on the ring. We use an aperture of a diameter 0." 47, corresponding to the PSF size of the $\mathrm{Ks}_{\mathrm{tot}}$ image. We then derive the colour differences of the nucleus and the ring

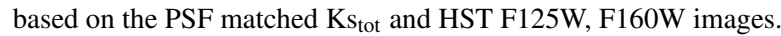

We find that the nucleus is 0.29 mag redder ( $3.6 \sigma$ significance) than the average colour of the ring in $\mathrm{F} 160 \mathrm{~W}-\mathrm{Ks}_{\mathrm{tot}}$, and 0.42 mag redder $(3.9 \sigma \mathrm{sig}-$ nificance) in F125W-F160W. The intrinsic colour difference might be larger without the beam-smearing of the PSF. For example, using the HST original images (i.e., without convolving with the PSF of the $\mathrm{Ks}_{\text {tot }}$ band), the nucleus is 0.54 mag redder ( $5.0 \sigma$ significance) than the ring in F125W-F160W. The redder colour of the "nucleus" region is consistent with the existence of an off-centre nucleus commonly seen in CRGs ${ }^{4}$. Well-known examples of CRGs with an off-centre nucleus are Arp 147 and NGC 985. Future highresolution images in the optical and near-infrared bands are required to further confirm the location of this nucleus.

\section{Star Formation Rate}

4.1 Total star formation rate The SFRs for ZFOURGE sources 49 are based on the combined rest-frame infrared luminosity $\left(\mathrm{L}_{\mathrm{IR}, 8-1000 \mu \mathrm{m}}\right)$ and rest-frame UV luminosity $\left(\mathrm{L}_{\mathrm{UV}, 1216-3000 \AA}\right)$. The $\mathrm{UV}+\mathrm{IR}$ approach assumes that the IR emission of galaxies comes from dust heated by the UV light of massive stars ${ }^{49}$. $\mathrm{L}_{\mathrm{IR}}$ is measured on an IR spectral template fitted to the 24 , 100 and $160 \mu \mathrm{m}$ far-IR photometry. The far-IR photometry is measured using apertures of $3^{\prime \prime}-6^{\prime \prime}$ from the MIPS/PACS imaging with the de-blending technique ${ }^{53}$. $\mathrm{L}_{U V}$ is measured on the EAzY photometric model ${ }^{49}$. The $\mathrm{SFR}_{\mathrm{UV}+\mathrm{IR}}$ for R5519 is calculated to be $80 \pm 0.2 \mathrm{M}_{\odot} \mathrm{yr}^{-1}$. Note that because of the proximity of the neighbouring galaxy G5593 and the large PSF $\left(\right.$ FWHM $\left.>4^{\prime \prime}\right)$ of the MIPS and PACS images, the main uncertainties in the SFR $_{\mathrm{UV}+\mathrm{IR}}$ of R5519 and G5593 come from the systematics of de-blending the two sources. G5593 is at a similar redshift as R5519 and is a bright nonAGN source detected in the $24 \mu \mathrm{m}$ with $\mathrm{SFR}_{\mathrm{UV}+\mathrm{IR}}=123 \pm 2 \mathrm{M}_{\odot} \mathrm{yr}^{-1}$. G5475 is a quiescent galaxy, consistent with its early-type morphology.

For comparison, we determine the dust-uncorrected SFR from the total $\mathrm{H} \alpha$ flux of the MOSFIRE slit spectrum and have $\mathrm{SFR}_{\mathrm{H} \alpha \text { (slit;with dust })}=3.7$ $\pm 0.1 M_{\odot} \mathrm{yr}^{-1}$. For dust attenuation correction on $\mathrm{H} \alpha(\mathrm{A}(\mathrm{H} \alpha))$, we infer from the stellar dust attenuation obtained via SED fitting $\left(\mathrm{A}_{\mathrm{v}, \mathrm{star}} \approx 1.1\right)$ We test two methods on dust correction. We first use the empirical relation of $\mathrm{A}(\mathrm{H} \alpha)$ ) and $\mathrm{A}_{\mathrm{v}, \text { star }}$ for $z \approx 2$ star-forming galaxies as a function of SFR(SED) and $M_{*}(\mathrm{SED}) \sqrt{54}$; we have $\left.\mathrm{A}(\mathrm{H} \alpha)\right)=0.21+\mathrm{A}_{\mathrm{v}, \mathrm{star}}$. The dust corrected $\mathrm{SFR}_{\mathrm{H} \alpha(\mathrm{slit})}$ is therefore $12.4 \pm 0.3 M_{\odot} \mathrm{yr}^{-1}$ for the first method. For the second method, we use the classic nebular attenuation curve ${ }^{55}$ with $R_{\mathrm{v}}=3.1$ and have $\mathrm{A}(\mathrm{H} \alpha)=2.53 \times \mathrm{E}(\mathrm{B}-\mathrm{V})_{\mathrm{HII}}$; Assuming $\mathrm{E}(\mathrm{B}-\mathrm{V})_{\text {star }}=0.44 \times$ $\mathrm{E}(\mathrm{B}-\mathrm{V})_{\mathrm{HII}}\left[52\right.$, we have $\mathrm{A}(\mathrm{H} \alpha)=1.42 \times \mathrm{A}_{\mathrm{v}, \text { star }}$ or $\mathrm{A}(\mathrm{H} \alpha)=1.42 \times \mathrm{A}_{\mathrm{v}, \mathrm{star}} 56$ [57. The dust corrected $\mathrm{SFR}_{\mathrm{H} \alpha(\text { slit })}$ is therefore $15.6 \pm 0.4 M_{\odot} \mathrm{yr}^{-1}$ for the second method. Finally, we estimate the slit loss factor by aligning the MOSFIRE slit on the PSF matched $\mathrm{H} \alpha$ narrow-band image and calculating the fraction of flux that is outside of the slit on the $\mathrm{H} \alpha$ narrow-band image. We derive a slit-loss factor of $3.1 \pm 1.1$. The final slit-loss and dust attenuation corrected $\mathrm{SFR}_{\mathrm{H} \alpha}$ is $38.4 \pm 13.6 M_{\odot} \mathrm{yr}^{-1}$ for the first method and $48.4 \pm 17.2 M_{\odot} \mathrm{yr}^{-1}$ for the second method, with errors representing statistical errors contributed mainly by the slit-loss correction uncertainty. Though the H $\alpha$ SFR is $\sim 2 \times$ smaller than the $\mathrm{SFR}_{\mathrm{UV}+\mathrm{IR}}$, the discrepancy is not surprising given the large uncertainty in dust attenuation and slit loss.

4.2 Spatial distribution and $\Sigma_{\mathrm{SFR}}$ Using the location of the best fit double ellipse and the $\mathrm{H} \alpha$ narrow band image, we estimate that $\sim 45-70 \%$ of the star formation occurs on the ring. The upper limit is calculated by assuming that the $\mathrm{H} \alpha$ narrow band image traces all recent star formation activity. The lower limit is estimated by including only $\mathrm{H} \alpha$ pixels within the ring that are more than $5 \sigma$ brighter than the average $\mathrm{H} \alpha$ surface brightness. We caution that this estimation does not include uncertainties from the beam-smearing of the $\mathrm{H} \alpha$ narrow band image, spatial variation of the dust attenuation, and the contribution of faint $\mathrm{SF}$ regions.

Our current data do not have the spatial resolution and SNR to calculate the spatially resolved $\Sigma_{\mathrm{SFR}}$. We provide two simple estimates for an average $\Sigma_{\mathrm{SFR}}$. We first use $\mathrm{SFR}_{\mathrm{UV}+\mathrm{IR}}$ divided by the circular area within $\mathrm{R}_{80}$ and have $\Sigma_{\mathrm{SFR}} \sim 0.2 \mathrm{M}_{\odot} \mathrm{yr}^{-1} \mathrm{kpc}^{-1}$. We then use $\mathrm{SFR}_{\mathrm{H} \alpha}=38 \mathrm{M}_{\odot} \mathrm{yr}^{-1}$ divided by the area of the ring defined by the best-fit ellipse and have $\Sigma_{\mathrm{SFR}}$ $\sim 0.4 \mathrm{M}_{\odot} \mathrm{yr}^{-1} \mathrm{kpc}^{-1}$.

4.3 Comparing with local CRGs on the $\mathrm{M}_{*}$-SFR relation The wellestablished correlation between SFR and stellar mass $M_{*}$ is the so-called "main-sequence" for star-forming galaxies. The slope and scatter of the $\mathrm{M}_{*-}$ SFR relation does not evolve significantly from $z \sim 0$ to $z \approx 2$ [ref. $\left.{ }^{[28}\right]$. At at a fixed stellar mass, star-forming galaxies at $z \sim 2$ have $\sim 20$ times higher SFR compared with $z \sim 0$ star-forming galaxies. Based on $\mathrm{SFR}_{\mathrm{UV}+\mathrm{IR}}, \mathrm{R} 5519$ is a star-forming galaxy that lies within the $1 \sigma$ scatter $(\sim 0.3 \mathrm{dex})$ of the $\mathrm{M}_{*}-\mathrm{SFR}$ relation at $z \approx 2[\mathrm{ref} \cdot \overline{49}]$.

Local CRGs have moderately higher SFR than other local spirals. To compare R5519 with local CRGs on the $\mathrm{M}_{*}$-SFR relation, we use a sample of local CRGs 25 that have both the SFR and stellar mass measured in a selfconsistent way. We exclude two CRGs that have contaminations in their SFR from either a neighbouring galaxy or an AGN. We also include literature data for Arp 147 [ref. $[27]$ and the Cartwheel galaxy $\sqrt{58}$, as well as our Milky Way 59 . As there is no reported stellar mass for the Cartwheel galaxy, we scale its dynamical mass to a stellar mass using the scaling relation of local galaxies $^{60}$ (Supplementary Fig.11).

Data Availability The imaging data presented here are publicly available from the ZFOURGE survey website (http://z fourge.tamu.edu/l and from the 3DHST archive https://archive.stsci.edu/prepds/3d-hst//. The spectroscopic data of this work was based on observations made with the Keck telescope from the W. M. Keck Observatory. The raw spectroscopic data can be accessed through the publicly available Keck Observatory Archive (https://www2. keck.hawaii.edu/koa/public/koa.php. The reduced data and other data that support the plots within this paper and other findings of this study are available from the corresponding author on reasonable request.

Code availability The customised MOSFIRE spectroscopic fitting code used in this work can be found here http://astronomy.swin.edu.au/ tyuan/ mosfit/. Scripts related to EAGLE simulations analysis in this paper are available from the second author (A.E., email: ahmedagali70@gmail.com) on reasonable request. Other scripts related to the analysis in this paper are available from the corresponding author (T.Y.) on reasonable request.

Supplementary information accompanies this paper. The author's link to the Supplementary Information (SI) can be found here http://astronomy.swin. edu.au/ tyuan/paper/ The SI includes 10 sections, 16 figures and 4 tables.

\section{References}


31. Planck Collaboration XVI et al. Planck 2013 results. XVI. Cosmological parameters. Astron. Astrophys. 571, 16 (2014).

32. Oke, J. B. \& Gunn, J. E. Secondary standard stars for absolute spectrophotometry. Astrophys. J. 266, 713-717 (1983).

33. Grogin, N. A. et al. CANDELS: The Cosmic Assembly Near-infrared Deep Extragalactic Legacy Survey. Astrophys. J. Supp. 197, 35 (2011).

34. Skelton, R. E. et al. 3D-HST WFC3-selected Photometric Catalogs in the Five CANDELS/3D-HST Fields: Photometry, Photometric Redshifts, and Stellar Masses. Astrophys. J. Supp. 214, 24 (2014).

35. Miller, T. B. Gunn, J. E. van Dokkum, P. Mowla, L. \& van der Wel, A. A New View of the Size-Mass Distribution of Galaxies: Using $r_{20}$ and $r_{80}$ Instead of $\mathrm{r}_{50}$. Astrophys. J. 872, L14 (2019).

36. Graham, A. W. \& Driver, S. P. A Concise Reference to (Projected) Srsic R $\mathrm{R}^{1 / n}$ Quantities, Including Concentration, Profile Slopes, Petrosian Indices, and Kron Magnitudes. Publications of the Astronomical Society of Australia 22, 118-127 (2005)

37. de Vaucouleurs, G. et al. Third Reference Catalogue of Bright Galaxies (RC3). Springer-Verlag, New York, (1991).

38. Mowla, L. van der Wel, A. van Dokkum, P. \& Miller, T. B. A Mass-dependent Slope of the Galaxy Size-Mass Relation out to $z \sim 3$ : Further Evidence for a Direct Relation between Median Galaxy Size and Median Halo Mass. Astrophys. J. 872, L13 (2019).

39. Juric, M. et al. The Milky Way Tomography with SDSS. I. Stellar Number Density Distribution. Astrophys. J. 673, 864-914 (2008).

40. Wegg, C. Gerhard, O. \& Portail, M. The structure of the Milky Way's bar outside the bulge. Mon. Not. R. Astron. Soc. 450, 4050-4069 (2015).

41. Bland-Hawthorn, J. \& Gerhard, O. The Galaxy in Context: Structural, Kinematic, and Integrated Properties. ARA\&A 54, 529-596 (2016).

42. Glazebrook, K. The Dawes Review 1: Kinematic Studies of Star-Forming Galaxies Across Cosmic Time. Publications of the Astronomical Society of Australia 30, 056 (2013).

43. Giovanelli, R. On the scaling relations of disk galaxies. IAU Symposium 289 296-303 (2013).

44. Few, M. A., Madore, B. F. \& Arp, H. C. Ring galaxies. I - Kinematics of the southern ring galaxy AM 064-741. Mon. Not. R. Astron. Soc. 199, 633-647 (1982).

45. Higdon, J. L. Wheels of Fire. II. Neutral Hydrogen in the Cartwheel Ring Galaxy. Astrophys. J. 467, 241 (1996).

46. Higdon, J. L. Higdon, S. J. U. \& Rand, R. J. Wheels of Fire. IV. Star Formation and the Neutral Interstellar Medium in the Ring Galaxy AM0644-741. Astrophys. J. 739, 97 (2011).

47. Kriek, M. et al. An Ultra-Deep Near-Infrared Spectrum of a Compact Quiescent Galaxy at $z=2.2$. Astrophys. J. 700, 221-231 (2009).

48. Brammer, G. B. van Dokkum, P. G. \& Coppi, P. EAZY: A Fast, Public Photometric Redshift Code. Astrophys. J. 686, 1503-1513 (2008).

49. Tomczak, A. R. et al. The SFR-M* Relation and Empirical Star-Formation Histories from ZFOURGE at $0.5<z<4$. Astrophys. J. 817, 118 (2016).

50. Bruzual, G. \& Charlot, S. Stellar population synthesis at the resolution of 2003. Mon. Not. R. Astron. Soc. 344, 1000-1028 (2003).

51. Chabrier, G. Galactic Stellar and Substellar Initial Mass Function. PASP 115, 763-795 (2003).

52. Calzetti, D. et al. The Dust Content and Opacity of Actively Star-forming Galaxies. Astrophys. J. 533, 682-695 (2000).

53. Labbe, I. et al. Spitzer IRAC Confirmation of z850-Dropout Galaxies in the Hubble Ultra Deep Field: Stellar Masses and Ages at $z \sim 7$. Astrophys. J. 649, L67-L70 (2006).

54. Reddy, N. A. et al. The MOSDEF Survey: Measurements of Balmer Decrements and the Dust Attenuation Curve at Redshifts $z 1.4-2.6$. Astrophys. J. 806, 259 (2015).

55. Cardelli, J. A. Clayton, G. C. \& Mathis, J. S. The relationship between infrared, optical, and ultraviolet extinction. Astrophys. J. 345, 245-256 (1989).

56. Steidel, C. C. et al. Strong Nebular Line Ratios in the Spectra of $z \sim 2-3$ Star Forming Galaxies: First Results from KBSS-MOSFIRE. Astrophys. J. 795, 165 (2014).

57. Tran, K. V. H. et al. ZFIRE: Galaxy Cluster Kinematics, H alpha Star Formation Rates, and Gas Phase Metallicities of XMM-LSS J02182-05102 at $z c l=1.6232$. Astrophys. J. 811, 28 (2015).

58. Crivellari09, E. ,Wolter, A. \& Trinchieri, G. The Cartwheel galaxy with XMMNewton. Astron. Astrophys. 501, 445-453 (2009).

59. Licquia, T. C. \& Newman, J. A. Improved Estimates of the Milky Way's Stellar Mass and Star Formation Rate from Hierarchical Bayesian Meta-Analysis. Astrophys. J. 806, 96 (2015)

60. Taylor, E. N. et al. On the Masses of Galaxies in the Local Universe. Astrophys. J. 722, 1-19 (2010). 